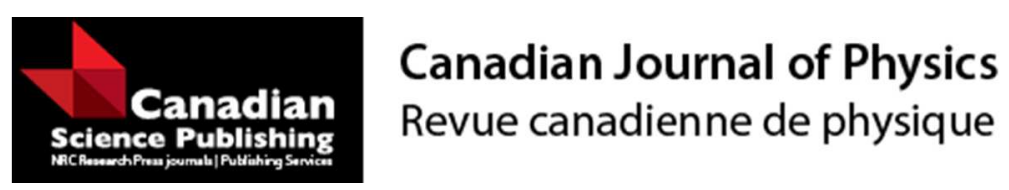

\title{
Chaotic inflation in spatially homogeneous Bianchi type V space-time
}

\begin{tabular}{|r|l|}
\hline Journal: & Canadian Journal of Physics \\
\hline Manuscript ID & cjp-2017-0163.R1 \\
\hline Manuscript Type: & Article \\
\hline Date Submitted by the Author: & 03-Apr-2017 \\
\hline Complete List of Authors: & $\begin{array}{l}\text { Bali, Raj; University of Rajasthan, Mathematics } \\
\text { Kumari, Parmit; University of Rajasthan, Department of Mathematics }\end{array}$ \\
\hline $\begin{array}{r}\text { Keyword: } \\
\text { Is the invited manuscript for } \\
\text { consideration in a Special } \\
\text { Issue? : }\end{array}$ & N/A \\
\hline \multicolumn{2}{|l}{} \\
\hline
\end{tabular}




\title{
Chaotic inflation in spatially homogeneous Bianchi type V space-time Raj Bali ${ }^{\mathrm{a}}$ and P. Kumari ${ }^{\mathrm{b}}$
}

\begin{abstract}
Chaotic inflationary scenario in spatially homogeneous Bianchi type V space-time following Linde [9] and the condition $\mathrm{R}^{3}=e^{3 H_{0} t}$ based on theory of super cooling during the cosmological phase transition proposed by Kirzhnits \& Linde [10], is discussed. It has been found that the model represents anisotropic phase of Universe in general but at late time, it isotropizes. The deceleration parameter $(\mathrm{q})=-1$ indicates that the model leads to de-Sitter space time. It is found that inflationary parameters viz. slow roll parameters, and anisotropic parameters are in excellent agreement with Planck 2013 results [11].
\end{abstract}

Keywords: Chaotic, inflation, spatially, homogeneous, Bianchi type V

\section{Introduction}

Bianchi type $\mathrm{V}$ universes create more interest in the study because these are anisotropic generalization of open FRW models and have arbitrary small anisotropy levels at any instant of cosmic time. A number of authors viz. Collins[1], Roy and Singh[2], Banerjee and Sanyal[3], Ram[4], Coley[5], Bali and Singh[6], Bali and Kumawat[7], Singh[8] have studied Bianchi type V cosmological models in different contexts.

On the basis of available observations (CMB and abundance of light elements), it was believed that Universe was created in hot big bang. Therefore, it was so difficult to overcome a certain psychological barrier and abandon all these assumptions. This was resolved by the invention of chaotic inflation scenario as proposed by Linde [9]. According to this scenario, inflation may begin even if there was no thermal equilibrium in the early Universe and it may occur in the theories with simplest potential $\mathrm{V}(\phi) \sim \phi^{2}$ where $\phi$ is scalar field. Chaotic inflation occurs when the potential has flat region which allows the existence of 
slow roll region. Linde [9] proposed chaotic universe with an assumption that the present universe is originated from a chaotic distribution of scalar field when potential energy of field dominates over kinetic energy. Later, it has been shown by Bunn et al. [13] that chaotic scenario can be realized even when scalar field is kinetic energy dominated. Paul et al. [14] have pointed out that Linde's chaotic inflationary scenario is fairly general and can be accommodated even if the universe is anisotropic. Linde's first model [9] of chaotic inflation was based on polynomial potential $\mathrm{V}(\phi)=\frac{1}{2} m^{2} \phi^{2}+\frac{\lambda}{4} \phi^{4}$, where $\phi$ is scalar field, $\lambda$ is a constant. Recently Bali [15] investigated chaotic inflationary universe scenario in Bianchi type I space-time assuming $\mathrm{R}^{3}=e^{3 H_{o} t}$ and $\mathrm{V}(\phi)=\frac{1}{2} m^{2} \phi^{2}+\frac{\lambda}{n} \phi^{n} \quad$ following Linde $[9,12]$ assuming $\ddot{\phi} \ll \frac{d V}{d \phi}$.

In the present investigation, we have investigated chaotic inflationary scenario in Bianchi Type V space-time assuming

$\mathrm{V}(\phi)=\frac{1}{2} m^{2} \phi^{2}+\frac{\lambda}{n} \phi^{n}, \lambda$ being a constant. To get deterministc scenario, we also assume that $\mathrm{R}^{3}=e^{3 H_{0} t}$ as proposed by Kirzhnits and Linde [10] where $\mathrm{R}$ is scale factor. We find that inflationary parameters are in excellent agreement with Planck 2013 results [11].

\section{Metric and field equations}

Bianchi Type V space-time is the natural generalization of FRW (FriedmannRobertson-Walker) model with negative curvature. This open model is favoured by the available evidences for low density universes (Gott. et al. [16]). These models contain isotropy special cases and permit arbitrarily small anisotropy levels at 
some instant of cosmic time. These properties make them suitable as models of our universe.

We consider Bianchi Type V space-time in orthogonal form as

$d s^{2}=-d t^{2}+A^{2} d x^{2}+e^{2 x}\left(B^{2} d y^{2}+C^{2} d z^{2}\right)$

where A,B,C are functions of $\mathrm{t}$-alone.

The energy-momentum tensor with scalar field $(\phi)$ is given by

$$
\mathrm{T}_{\mathrm{i}}^{\mathrm{j}}=\partial_{\mathrm{i}} \phi \partial^{\mathrm{j}} \phi-\left\{\frac{1}{2} \partial_{\mathrm{k}} \phi \partial^{\mathrm{k}} \phi+\mathrm{V}(\phi)\right\} \mathrm{g}_{\mathrm{i}}^{\mathrm{j}}
$$

The Lagrangian in which gravity is minimally coupled to scalar field $(\phi)$ is defined by Stein-Schabes [17] as

$$
\mathrm{L}=\int_{M}\left[\sqrt{-g} R_{i}^{j}-\frac{1}{2} g^{i j} \partial_{i} \phi \partial_{j} \phi-V(\phi)\right] d^{4} x
$$

The energy conservation law coincides with equation of motion for scalar field $(\phi)$ and we have

$$
\frac{1}{\sqrt{-\mathrm{g}}} \partial_{\mathrm{i}}\left(\sqrt{-\mathrm{g}} \partial^{\mathrm{i}} \phi\right)=-\frac{\mathrm{dV}}{\mathrm{d} \phi}
$$

which leads to

$$
\phi_{44}+\phi_{4}\left(\frac{\mathrm{A}_{4}}{\mathrm{~A}}+\frac{\mathrm{B}_{4}}{\mathrm{~B}}+\frac{\mathrm{C}_{4}}{\mathrm{C}}\right)=\frac{\mathrm{dV}}{\mathrm{d} \phi}
$$

We assume the coordinates to be co-moving so that $\mathrm{v}^{1}=0=\mathrm{v}^{2}=\mathrm{v}^{3}, \mathrm{v}^{4}=1$.

The Einstein's field equation

$\mathrm{R}_{\mathrm{i}}^{\mathrm{j}}-\frac{1}{2} \mathrm{Rg}_{\mathrm{i}}^{\mathrm{j}}=-\mathrm{T}_{\mathrm{i}}^{\mathrm{j}}$ 
(in gravitational units $8 \pi \mathrm{G}=\mathrm{c}=1$ )

for the line-element (1), leads to

$\frac{\mathrm{B}_{44}}{\mathrm{~B}}+\frac{\mathrm{C}_{44}}{\mathrm{C}}+\frac{\mathrm{B}_{4} \mathrm{C}_{4}}{\mathrm{BC}}-\frac{1}{\mathrm{~A}^{2}}=-\frac{1}{2} \phi_{4}^{2}+\mathrm{V}(\phi)$

$\frac{\mathrm{A}_{44}}{\mathrm{~A}}+\frac{\mathrm{C}_{44}}{\mathrm{C}}+\frac{\mathrm{A}_{4} \mathrm{C}_{4}}{\mathrm{AC}}-\frac{1}{\mathrm{~A}^{2}}=-\frac{1}{2} \phi_{4}^{2}+\mathrm{V}(\phi)$

$\frac{\mathrm{A}_{44}}{\mathrm{~A}}+\frac{\mathrm{B}_{44}}{\mathrm{~B}}+\frac{\mathrm{A}_{4} \mathrm{~B}_{4}}{\mathrm{AB}}-\frac{1}{\mathrm{~A}^{2}}=-\frac{1}{2} \phi_{4}^{2}+\mathrm{V}(\phi)$

$\frac{\mathrm{A}_{4} \mathrm{~B}_{4}}{\mathrm{AB}}+\frac{\mathrm{B}_{4} \mathrm{C}_{4}}{\mathrm{BC}}+\frac{\mathrm{A}_{4} \mathrm{C}_{4}}{\mathrm{AC}}-\frac{3}{\mathrm{~A}^{2}}=\frac{1}{2} \phi_{4}^{2}+\mathrm{V}(\phi)$

$\frac{2 \mathrm{~A}_{4}}{\mathrm{~A}}-\frac{\mathrm{B}_{4}}{\mathrm{~B}}-\frac{\mathrm{C}_{4}}{\mathrm{C}}=0$

Equation (10) leads to

$$
\mathrm{A}^{2}=\mathrm{BC}
$$

where constant of integration is taken as unity for simplicity.

\section{Solution of field equations}

Equations (7) and (8) lead to

$$
\frac{\mathrm{B}_{44}}{\mathrm{~B}}-\frac{\mathrm{C}_{44}}{\mathrm{C}}+\frac{\mathrm{A}_{4}}{\mathrm{~A}}\left(\frac{\mathrm{B}_{4}}{\mathrm{~B}}-\frac{\mathrm{C}_{4}}{\mathrm{C}}\right)=0
$$

Using result (11) in equation (12), we have

$$
\frac{\mathrm{B}_{44}}{\mathrm{~B}}-\frac{\mathrm{C}_{44}}{\mathrm{C}}+\frac{1}{2}\left(\frac{\mathrm{B}_{4}}{\mathrm{~B}}+\frac{\mathrm{C}_{4}}{\mathrm{C}}\right)\left(\frac{\mathrm{B}_{4}}{\mathrm{~B}}-\frac{\mathrm{C}_{4}}{\mathrm{C}}\right)=0
$$

which leads to 
$\frac{\left(\mathrm{CB}_{4}-\mathrm{BC}_{4}\right)_{4}}{\left(\mathrm{CB}_{4}-\mathrm{BC}_{4}\right)}=-\frac{1}{2}\left(\frac{\mathrm{B}_{4}}{\mathrm{~B}}+\frac{\mathrm{C}_{4}}{\mathrm{C}}\right)$

Thus, we have

$\mathrm{C}^{2}\left(\frac{\mathrm{B}}{\mathrm{C}}\right)_{4}=\frac{\mathrm{L}}{(\mathrm{BC})^{\frac{1}{2}}}$

where $\mathrm{L}$ is constant of integration. Equation (13) leads to

$$
\frac{v_{4}}{v}=\frac{L}{\mu^{\frac{3}{2}}}
$$

where $\mathrm{BC}=\mu$ and $\frac{\mathrm{B}}{\mathrm{C}}=v$

To get the deterministic model of our universe, we assume that

$$
\mathrm{R}^{3}=\mathrm{ABC}=e^{3 H_{0} t}
$$

where $\mathrm{H}_{0}$ is Hubble constant.

Thus, we have

$\frac{\mathrm{A}_{4}}{\mathrm{~A}}+\frac{\mathrm{B}_{4}}{\mathrm{~B}}+\frac{\mathrm{C}_{4}}{\mathrm{C}}=3 \mathrm{H}_{0}$

Using (10) in equation (16), we have

$\frac{\mathrm{B}_{4}}{\mathrm{~B}}+\frac{\mathrm{C}_{4}}{\mathrm{C}}=2 \mathrm{H}_{0}$

which leads to

$\frac{\mu_{4}}{\mu}=2 \mathrm{H}_{0}$ 
Equation (17) leads to

$$
\mu=\mathrm{Ne}^{2 \mathrm{H}_{0}{ }^{\mathrm{t}}}
$$

$\mathrm{N}$ being constant of integration.

From equations (14) and (18), we have

$$
v=M \exp \left\{\frac{-\mathrm{L}}{3 \mathrm{H}_{0} \mathrm{~N}^{\frac{3}{2}}} \mathrm{e}^{-3 \mathrm{H}_{0}{ }^{\mathrm{t}}}\right\}
$$

Thus

$$
\begin{aligned}
& \mathrm{B}^{2}=\mu \nu=M \mathrm{MN}^{2 \mathrm{H}_{0}{ }^{t}} \exp \left\{\frac{-\mathrm{L}}{3 \mathrm{H}_{0} \mathrm{~N}^{\frac{3}{2}}} \mathrm{e}^{-3 \mathrm{H}_{0}{ }^{t}}\right\} \\
& \mathrm{C}^{2}=\frac{\mu}{v}=\frac{\mathrm{Ne}^{2 \mathrm{H}_{0} t^{t}}}{\operatorname{Mexp}\left\{\frac{-\mathrm{L}}{3 \mathrm{H}_{0} \mathrm{~N}^{\frac{3}{2}}} \mathrm{e}^{-3 \mathrm{H}_{0} t^{t}}\right\}}
\end{aligned}
$$

$$
\mathrm{A}^{2}=\mathrm{BC}=\mu=\mathrm{Ne}^{2 \mathrm{H}_{0}{ }^{t}}
$$

After suitable transformation of coordinates, the metric (1) leads to the form

$$
d s^{2}=-d T^{2}+e^{2 H_{0}^{T}} d X^{2}+e^{\frac{2 x}{N}}\left\{e^{2 H_{0}^{T}} \exp \left(\frac{-\mathrm{L}^{-3 H_{0}^{T}}}{3 H_{0} N^{\frac{3}{2}}}\right) d Y^{2}+\frac{e^{2 H_{0}{ }^{T}}}{\exp \left\{\frac{-L^{2}}{3 H_{0} N^{\frac{3}{2}}} e^{-3 H_{0}^{T}}\right\}} d Z^{2}\right\}
$$

where

$$
\sqrt{\mathrm{N}} \mathrm{x}=\mathrm{X}, \sqrt{\mathrm{MN}} \mathrm{y}=\mathrm{Y}, \sqrt{\frac{\mathrm{N}}{\mathrm{M}}} \mathrm{z}=\mathrm{Z}, \mathrm{t}=\mathrm{T}
$$


To find the value of scalar field $(\phi)$ :

For chaotic inflation, we assume that potential $\mathrm{V}(\phi)$ is given by

$\mathrm{V}(\phi)=\frac{1}{2} m^{2} \phi^{2}+\frac{\lambda}{n} \phi^{n}$

where $m, n, \lambda$ are constants and $\lambda>0$.

Following Linde [9] for chaotic inflation, we consider

$\phi_{44}<<\frac{d V}{d \phi}$

Using (25) in equation (4), we have

$\frac{\mathrm{d} \phi}{\phi\left[\mathrm{m}^{2}+\lambda \phi^{\mathrm{n}-2}\right]}=\frac{1}{3 \mathrm{H}_{0}} \mathrm{dt}$

which leads to

$\frac{\phi^{\mathrm{n}-3} d \phi}{\phi^{\mathrm{n}-2}\left[\mathrm{~m}^{2}+\lambda \phi^{\mathrm{n}-2}\right]}=\frac{1}{3 \mathrm{H}_{0}} \mathrm{dt}$

Thus, we have

$\phi^{n-2}=\frac{b l}{e^{-a t}-1}$

where $\mathrm{a}=\frac{\lambda(\mathrm{n}-2) \mathrm{b}}{3 \mathrm{H}_{0}}$, and $l$ is constant of integration.

\section{Physical and geometrical feature}

The spatial volume $\left(\mathrm{R}^{3}\right)$, the expansion $(\theta)$, shear $(\sigma)$, Hubble parameter $(\mathrm{H})$, the deceleration parameter $(q)$ for the model (23) are given by

$\mathrm{R}^{3}=e^{3 H_{0} t}$ 
$\theta=\frac{\mathrm{A}_{4}}{\mathrm{~A}}+\frac{\mathrm{B}_{4}}{\mathrm{~B}}+\frac{\mathrm{C}_{4}}{\mathrm{C}}=3 \mathrm{H}_{0}$

$\sigma=\frac{1}{2}\left(\frac{\mathrm{B}_{4}}{\mathrm{~B}}-\frac{\mathrm{C}_{4}}{\mathrm{C}}\right)=\frac{\mathrm{L}}{\mathrm{N}^{\frac{3}{2}}} \mathrm{e}^{-3 \mathrm{H}_{0}{ }^{t}}$

$H=\frac{R_{4}}{R}=H_{0}$

$\frac{\sigma}{\theta}=\frac{\mathrm{Le}^{-3 \mathrm{H}_{0}{ }^{t}}}{3 \mathrm{H}_{0} \mathrm{~N}^{\frac{3}{2}}}$

$\mathrm{q}=\frac{-\mathrm{R}_{44} / \mathrm{R}}{\mathrm{R}_{4}^{2} / \mathrm{R}^{2}}=-1$

Now, we calculate inflationary parameters i.e., slow roll parameters $(\varepsilon$,$) ,$ anisotropic parameter for the model (23) to examine whether these parameters are in excellent agreement with Planck 2013 results. For the model (23), the scale factor $(R)$ is given by

$\mathrm{R}=\mathrm{e}^{\mathrm{H}_{0}^{\mathrm{t}}}$

The Hubble parameter $(\mathrm{H})$ is given by

$\mathrm{H}=\frac{\mathrm{R}_{4}}{\mathrm{R}}=\mathrm{H}_{0}$ (constant)

The slow roll parameter $(\varepsilon$,$) are defined as given by Unnikrishnan and Sahni [18]$

$\varepsilon=-\frac{\mathrm{H}_{4}}{\mathrm{H}^{2}}=0$

and

$\delta=\varepsilon-\frac{\varepsilon_{4}}{2 H \varepsilon}=0$ 
Slow roll parameters $(\varepsilon$, corresponds to $\varepsilon<<1, \delta<<1$.

It is convenient to assume the Lagrangianfor scalar field $\phi$ as

$\mathrm{L}(\phi, \mathrm{X})=\mathrm{X}-\mathrm{V}(\phi)$

where

$\mathrm{X}=\frac{\dot{\phi}^{2}}{2}$

For a generic $\mathrm{L}(\phi, \mathrm{X})$, it is convenient to introduce a third slow roll parameter $\mathrm{S}$ as given by $\mathrm{Hu}[19]$ :

$\mathrm{S}=\frac{\dot{\mathrm{C}}_{\mathrm{s}}}{\mathrm{HC}_{\mathrm{s}}}$

where $\mathrm{C}_{\mathrm{s}}$ is the speed of sound of the field as given by Garriga and Mukhanov [20]:

$\mathrm{C}_{\mathrm{s}}^{2}=\frac{\partial \mathrm{L} / \partial \mathrm{X}}{\frac{\partial \mathrm{L}}{\partial \mathrm{X}}+2 \mathrm{X} \frac{\partial^{2} \mathrm{~L}}{\partial \mathrm{X}^{2}}}=1$

Thus $\mathrm{S}=0$ requires not only $\varepsilon \ll 1, \delta \ll 1$ but also $|S| \ll 1$. For a canonical scalar field, the value of $\mathrm{S}$ is identically zero and this is also the case for kinematically driven as well as non-canonical model (Unnikrishnan et al.[21]).

\section{Anisotropic parameter}

The average anisotropic parameter $\left(\tilde{A}_{m}\right)$ is given by

$\tilde{\mathrm{A}}_{\mathrm{m}}=\frac{1}{3}\left[\sum_{\mathrm{i}=1}^{3}\left(\frac{\Delta \mathrm{H}_{\mathrm{i}}}{\mathrm{H}}\right)^{2}\right]$ 
Where $\mathrm{H}_{1}=\mathrm{H}_{1}-\mathrm{H}, \Delta \mathrm{H}_{2}=\mathrm{H}_{2}-\mathrm{H}, \Delta \mathrm{H}_{3}=\mathrm{H}_{3}-\mathrm{H}$ and $\mathrm{H}_{1}=\frac{\mathrm{A}_{4}}{\mathrm{~A}}, \mathrm{H}_{2}=\frac{\mathrm{B}_{4}}{\mathrm{~B}}, \mathrm{H}_{3}=\frac{\mathrm{C}_{4}}{\mathrm{C}}$.

Thus anisotropic parameter $\left(\tilde{A}_{m}\right)$ is given by

$$
\tilde{\mathrm{A}}_{\mathrm{m}}=\frac{1}{6} \frac{\mathrm{e}^{-6 \mathrm{H}_{0}}}{\mathrm{~N}^{3 \mathrm{H}_{0}^{2}}}
$$

\section{Discussion and conclusion}

The potential energy has minimum value at $\phi=0$. The scalar field $\phi$ at $\mathrm{t}=0$ is given by

$\phi^{n-2}=\frac{b l}{e^{-a t}-1}, 0<l<1$

The scalar field evolves slowly where $0<l<1$ and has maximum value at $l=1$ at $\mathrm{t}=0$. The model has constant expansion but accelerating phase. From equation (27), we find that $\phi^{\mathrm{n}-2}<1$ at $\mathrm{t}=0$ where $\mathrm{n} \geq 4 \& 0<l<1,0<\mathrm{b}<1$. Also $\mathrm{V}(\phi)=\frac{1}{2} m^{2} \phi^{2}+\frac{\lambda}{n} \phi^{n}<1$ as $\phi^{2}<1$ and $\mathrm{n} \geq 4$. Thus initial condition of inflation is satisfied. We also find that $\phi_{4}^{2}<\mathrm{V}(\phi)$. Therefore, chaotic inflationary scenario is realized for anisotropic Bianchi type V space-time. The spatial volume increases with time and average anisotropic parameter $\left(\tilde{A}_{m}\right)$ is not zero initially i.e., anisotropy is initially large but disappears for large value of $t$ i.e., the model isotropizes at late time. This result agrees with the result as pointed out by Rothman and Ellis [22], Jensen and Stein-Schabes [23]. The deceleration parameter $\mathrm{q}=-1$ which shows that model leads to de-Sitter space-time. The assumption $\mathrm{R}^{3}=\mathrm{ABC}=e^{3 H_{0} T}$ does not lead to FRW model immediately but for large value of $\mathrm{T}$, it leads to FRW model. Since for Bianchi Type V metric shear $(\sigma) \cong e^{-3 H_{0} T} \neq 0$ but for large value of T, $\sigma=0$. Thus for large values of T, it leads to FRW model because shear is zero in FRW model. The chaotic model 
leads to an inflationary phase which also helps in isotropization of universe. There is no initial singularity in the model [23].

\section{Acknowledgement}

The authors are thankful to the Reviewer and Associate Editor for their valuable suggestions.

\section{References:}

1. C.B. Collins. Comm. Math. Phys. 23. 1237 (1971).

2. $\quad$ S.R. Roy and J.P. Singh. Aust. J. Phys. 38. 763 (1985).

3. A. Banerjee and A.K. Sanyal. Gen. Relativ. Gravit. 20. 103 (1988).

4. S. Ram. Int. J. Theor. Phys. 29. 901 (1990).

5. A.A. Coley. Gen. Relativ. Gravit. 22.3 (1990a).

6. R. Bali and D.K. Singh. Astrophys. Space Sci. 300. 387 (2005).

7. R. Bali and P. Kumawat. Phys. Lett. B 665. 332 (2008).

8. C.P. Singh. Pramana-J. Phys. 72.429 (209).

9. A.D.Linde. Phys. Lett. B 129. 177 (1983).

10. D.A.Kirzhnits and A.D.Linde. Annals. Phys. 101. 195 (1976).

11. P.A.R.Ade et al. Planck 2013 results, axiv: 1303. 5082 (2013).

12. A.D.Linde. Class Quant.Gravity 18. 3275 (2001).

13. E.F.Bunn, A.R.Liddle and M.White. Phys. Rev. D 56 (1986) 5917R.

14. B.C.Paul, D.P.Datta and S.Mukherjee. Modern Phys. Lett. 1, (1986) 149. 
15. R.Bali. Modern Phys. Lett. A 27 (2012) 1250049.

16. J.R.Gott, J.E.Gunn, D.N.Schramn and B.M.Tinsley. Astrophys. J. 194 (1974) 543.

17. J.A. Stein-Schabes. Phys. Rev. D 35 (1987) 2345.

18. S. Unnikrishnan and V.Sahni. arxiv; 1305. 5260v2 (2013).

19. W.Hu. Phys. Rev. D 84 (2011) 027303.

20. J. Garriga and V.F.Mukhanov. Phys. Lett. B 458(1999) 219.

21. S. Unnikrishnan, V.Sahni and A. Toporensky. JCAP 1208 (2012) 018.

22. T. Rothman and G.F.R.Ellis. Phys. Lett. B 180 (1986) 9.

23. L.G. Jensen and J.A.Stein-Schabes. Phys. Rev. D 34 (1986) 931. 